\title{
Water hammer modeling for water networks via hyperbolic PDEs and switched DAEs
}

\author{
Rukhsana Kausar and Stephan Trenn
}

\begin{abstract}
In water distribution network instantaneous changes in valve and pump settings introduce jumps and sometimes impulses. In particular, a particular impulsive phenomenon which occurs due to sudden closing of valve is the so called water hammer. It is classically modeled as a system of hyperbolic partial differential equations (PDEs). We observed that under some suitable assumptions the PDEs usually used to describe water flows can be simplified to differential algebraic equations (DAEs). The idea is to model water hammer phenomenon in the switched DAEs framework due to its special feature of studying such impulsive effects. To compare these two modeling techniques, a system of hyperbolic PDE model and the switched DAE model for a simple set up consisting of two reservoirs, six pipes and three valve is presented. The aim of this contribution is to present results of both models as motivation for the claim that a switched DAE modeling framework is suitable for describing a water hammer.
\end{abstract}

\section{Introduction}

The occurrence of hydraulic transients in the operation of water distribution network is inevitable. Such transients are planned or accidental changes of the network configuration. These sudden structural changes can have dramatic effects in flow regimes, ranging from pump defects to catastrophic pipeline failures. The flow of water in pipes is usually model as system of nonlinear hyperbolic balance laws (i.e. partial differential equations, PDEs), see e.g. [4], where the sudden structural changes lead to large peaks and fast transients in the solution.

We propose to model such fast transients in the framework of switched differential algebraic equation (switched DAEs). This framework was originally introduced for modeling electrical circuits [12] and allows a precise mathematical description of peaks and fast transients in the form of Dirac impulses and jumps.

Fachbereich Mathematik, Technische Universität Kaiserslautern, Germany 
Our focus in this paper is on the so-called water hammer, which results from sudden changes of velocity in pipelines and can cause large pressures magnitudes. It is usually created by rapidly closing valves, shutting off or restarting pumps. Our goal is to show that these pressure peaks occurring in the PDE simulations can be well approximated by a suitable switched DAE model.

The paper is organized as follows. In Section 2 the water network and its components are defined as a graph and the mathematical models of the pipes and other components (like reservoir and valves) are introduced. In Section 3 we study in detail a simple water network which exhibits a water hammer; in particular, we derive the corresponding PDE model as well as a switched DAE model. In Section 4, we describe the solution theory used in solving our sample network problem. In Section 5 a numerical comparison of the PDE and switched DAE model are presented.

\section{Mathematical model}

The structure of a water network can be modeled as $G=(\mathbb{V}, \mathbb{E})$ where $\mathbb{V}$ is the set of nodes and $\mathbb{E} \subseteq \mathbb{V} \times \mathbb{V}$ is the set of edges. Each edge $e \in \mathbb{E}$ corresponds to a pipe of the water network and the nodes $v \in \mathbb{V}$ are the connections or endpoints of pipes, including junctions, pumps, valves, or reservoirs. We denote by $\gamma_{v}^{-}\left(\gamma_{v}^{+}\right)$the set of all indices of edges $e_{i} \in \mathbb{E}$ outgoing (ingoing) from (to) the node $v \in \mathbb{V}$; see Figure 1 for an illustration of this notation.

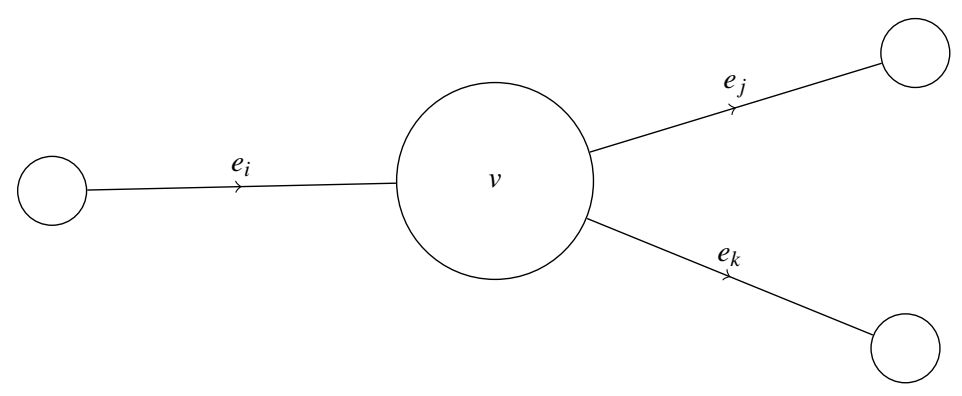

Fig. 1: A node $v$ with three incident edges $e_{i}, e_{j}, e_{k}$; here, $\gamma_{v}^{+}=\{i\}$ and $\gamma_{v}^{-}=\{j, k\}$.

In the model of water network elements, the two main physical quantities pressure and flow are involved. Those values at the end points of the pipes are related to each other corresponding to the type of node. Furthermore, the modeling of the flow in the pipes also involves density of the water. Usually, water is assumed to be incompressible, i.e. the density is assumed to be constant. However, our focus is on modeling the water hammer effect and for this it is necessary to take into account the (slight) compressibility of water. 


\subsection{Models of water flow in pipe}

One can model water flow in a pipe in two different ways depending on whether the compressibility of water is taken into account or not. In order to study transient phenomena like water hammer it is necessary to model compressibility, in particular, density and mass flow become space-dependent quantities. On the other hand, to understand the qualitative behavior, in particular, in large networks, it often suffices to model water as incompressible fluid. We will briefly introduce both models in the following.

\subsubsection{Compressible flow in a pipe}

Following [13] and [1] we use the following pressure law for compressible fluids:

$$
P(\rho)=P_{a}+K \frac{\rho-\rho_{a}}{\rho_{a}}
$$

where $K>0$ is the so called bulk modulus, $P_{a}>0$ is the atmospheric pressure and $\rho_{a}>0$ is the density at atmospheric pressure. The bulk modulus is related to the speed of sound $c>0$ as follows:

$$
c^{2}=\frac{\partial P}{\partial \rho}=K / \rho_{a}
$$

Note that $\beta:=1 / K$ is the so called compressibility coefficient. We consider a completely filled pipe of length $L>0$ with mass density $\rho(x, t)>0$ and mass flux $q(x, t) \in \mathbb{R}$ both defined on $[0, L] \times \mathbb{R}_{+}$. The compressible flow of water in the pipe can be modeled by the balance law of the following form [3, Sec. 2]:

$$
\begin{aligned}
\partial_{t} \rho+\partial_{x} q & =0 \\
\partial_{t} q+\partial_{x}\left(\frac{q^{2}}{\rho}+P(\rho)\right) & =-c_{f} \frac{q|q|}{2 D \rho},
\end{aligned}
$$

with the pressure law $P: \mathbb{R}_{+} \rightarrow \mathbb{R}_{+}$given by (1) and where $c_{f}>0$ is the friction against the pipe wall and $D>0$ is the diameter of the pipe. The initial condition for (3) is:

$$
q(x, 0)=q_{0}(x) \text { and } P(\rho(x, 0))=p_{0}(x) \quad x \in[0, L],
$$

for some initial flow $q_{0}:[0, L] \rightarrow \mathbb{R}$ and some initial pressure $p_{0}:[0, L] \rightarrow \mathbb{R}_{+}$. Note that the initial condition is given implicitly in terms of the pressure and not explicitly in terms of the density. The reason is that the pressure is the more relevant physical quantity, in particular, when the pipes are coupled with other water network elements. When the individual pipes are connected with other elements of the overall water distribution network, additional boundary and so called coupling condition will be imposed. 


\subsubsection{Coupling conditions at intersection nodes}

The balance law (3) has to be completed by intial, boundary and coupling conditions across the whole network. Suppose the initial data $P_{l}\left(\rho_{l}(x, 0)\right)=p_{l, 0}$ and $q_{l}(x, 0)=q_{l, 0}$ are given for each pipe $l$ in the network, where $\rho_{l}, q_{l}$ ans $P_{l}$ denote density, flow and pressure along each pipe edge $e_{l}$. Admissible boundaries must be chosen in accordance with the characteristics. Preservation of mass yields the coupling condition

$$
\sum_{l \in \gamma_{v}^{+}} q_{l}(L, t)=\sum_{l \in \gamma_{v}^{-}} q_{l}(0, t) .
$$

and consistency of pressure yields

$$
p_{i}(L, t)=p_{j}(0, t), \quad \forall i \in \gamma_{v}^{+}, \quad j \in \gamma_{v}^{-}, \quad \forall v \in \mathbb{V} .
$$

Condition (5) is an analogue of Kirchoff's current law for electrical circuits.

\subsubsection{Quasi stationary water flow model}

After some initial transient behaviour, the water flow in the pipe may be assumed to get stationary, i.e. the flow is location independent and we write $Q(t)=\frac{q(x, t)}{A}$ (mass flux is mass flow per unit area), where $A=\pi D^{2} / 4$ is the area of the pipe. Furthermore the density is assumed constant in space and time, i.e. $\rho(x, t)=\rho$ for $(x, t) \in[0, L] \times \mathbb{R}_{+}$and the pressure variable $p(x, t)$ is not coupled to the density via (1) anymore (in particular, water is considered incompressible). The remaining dynamical behavior in the variables $Q(t), P_{0}(t)=p(0, t)$ and $P_{L}(t)=p(L, t)$ can be described by the following ODE $[2,5,6]$ :

$$
\frac{d Q}{d t}+\frac{A}{L}\left(P_{L}-P_{0}\right)+\frac{c_{f} Q|Q|}{2 D A \rho_{a}}=0 .
$$

\subsection{Other network elements}

\subsubsection{Reservoir}

A reservoir is a node in the water network graph with arbitrary mass flow but with given pressure. For example if a node $v_{i}$ is designated as reservoir then pressure at this node will be set as constant. 


\subsubsection{Valve}

A valve is a control element which can be opened or closed and is located at one end of an edge. A closed valve here is modelled as a boundary condition at the corresponding end of the pipe in the form of a prescribed zero flow (instead of the corresponding pressure consistency (6)). As an example, assume $e_{i}, e_{j} \in \mathbb{E}$ are connected at junction node $v$, and a valve is located at the end of pipe $e_{j}$, then if the valve is open we just have the coupling conditions (5) and (6); in case the valve is closed instead of (6), we have the boundary condition $q_{j}(L, t)=0$ and hence, due to (5), also $q_{i}(0, t)=0$ (if more than two pipes are incident with $v$ than there may still be a non-zero-flow through the node even if the valve is closed).

\section{Analysis of a simple water network}

We want to study the water hammer effect on a simple water network consisting of two reservoirs located at nodes $v_{R_{1}}$ and $v_{R_{2}}$, with given pressure $p_{v_{R_{1}}}$ and $p_{v_{R_{2}}}$, respectively, and six pipes of each with length $L$. Three valves $V_{1}, V_{2}$ and $V_{3}$ are located at the end of pipes 4 and 5 and at the beginning of pipe 6 , respectively, as shown in Figure 2. We assume here that these three valves are opened and closed synchronously, the asynchronous case is ongoing research.

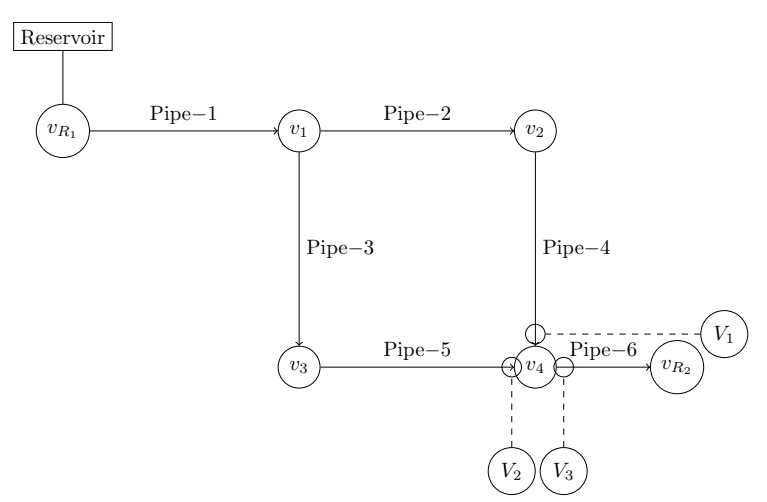

Fig. 2: Simple water network with two reservoirs at nodes $v_{R_{1}}$ and $v_{R_{2}}$, six pipes and three synchronous valves at node $v_{4}$. 


\subsection{PDE mode1}

Each pipe is modelled by system of balance laws given by (3) with pressure law (1) and for pipe $i$, will look as follows,

$$
\begin{aligned}
\partial_{t} \rho_{i}+\partial_{x} q_{i} & =0 \\
\partial_{t} q_{i}+\partial_{x}\left(\frac{q_{i}^{2}}{\rho_{i}}+P\left(\rho_{i}\right)\right) & =-c_{f_{i}} \frac{q_{i}\left|q_{i}\right|}{2 D_{i} \rho_{i}}
\end{aligned}
$$

For the sake of simplicity, we are using identical friction factors and diameters, i.e., $c_{f_{i}}=c_{f}, D_{i}=D, \forall i \in\{1, \cdots, 6\}$. Denote with $P_{i}(x, t)=P\left(\rho_{i}(x, t)\right)$ the pressure in the $i$-th pipe.

In contrast to [7], we present here a water hammer on a network with multiple valves so we need to take more coupling conditions into account: The vertices $v_{1}, v_{2}, v_{3}$ are coupling vertices and modeled by (6) and (5). At node $v_{4}$ valves are present at each incident pipe and it is assumed that they are initially open and simultaneously closed at $t=t_{S}$, resulting in the time-varying boundary condition:

$$
\left\{\begin{array}{lll}
(5),(6) \quad \text { at } \quad v_{4} & t \in\left(0, t_{S}\right) \\
q_{V_{1}}=q_{V_{2}}=q_{V_{3}}=0, & t>t_{S}
\end{array}\right.
$$

In the following the pressure at the valves is denoted by $p_{V_{1}}(t)=P_{4}(L, t), p_{V_{2}}(t)=$ $P_{5}(L, t)$ and $p_{V_{3}}(t)=P_{6}(0, t)$, respectively; moreover, $q_{V_{1}}(t)=q_{4}(L, t), q_{V_{2}}(t)=$ $q_{5}(L, t), q_{V_{3}}(t)=q_{6}(0, t)$.

\subsection{Switched DAE framework}

The quasi-stationary model (7) together with the corresponding coupling conditions for a setup as shown in Figure 2 leads to a switched DAE of the form,

$$
E_{\sigma} \dot{x}=A_{\sigma} x+f+g_{\sigma}(x)
$$

with $x=\left(Q_{1}, Q_{2}, Q_{3}, Q_{4}, Q_{5}, Q_{6}, P_{1}, P_{2}, P_{3}, P_{4}, P_{V_{1}}, P_{V_{2}}, P_{V_{3}}\right)^{\top}$ and

$$
\sigma(t)= \begin{cases}1, & t \in\left[0, t_{S}\right), \quad V_{1}, V_{2}, V_{3} \text { open } \\ 2, & t \geq t_{S}, \quad V_{1}, V_{2}, V_{3} \text { closed }\end{cases}
$$

The equations of the network when $t \in\left[0, t_{S}\right)$ are given as follows, 


$$
\begin{aligned}
-\frac{d Q_{1}}{d t}= & c_{1}\left(P_{v_{1}}-P_{R_{1}}\right)+c_{2} Q_{1}\left|Q_{1}\right|, \\
-\frac{d Q_{2}}{d t}= & c_{1}\left(P_{v_{2}}-P_{v_{1}}\right)+c_{2} Q_{2}\left|Q_{2}\right|, \\
-\frac{d Q_{3}}{d t}= & c_{1}\left(P_{v_{3}}-P_{v_{1}}\right)+c_{2} Q_{3}\left|Q_{3}\right|, \\
-\frac{d Q_{4}}{d t}= & c_{1}\left(P_{V_{1}}-P_{v_{2}}\right)+c_{2} Q_{4}\left|Q_{4}\right|, \\
-\frac{d Q_{5}}{d t}= & c_{1}\left(P_{V_{2}}-P_{v_{3}}\right)+c_{2} Q_{5}\left|Q_{5}\right|, \\
-\frac{d Q_{6}}{d t}= & c_{1}\left(P_{R_{2}}-P_{V_{3}}\right)+c_{2} Q_{6}\left|Q_{6}\right|, \\
& Q_{1}-Q_{2}-Q_{3}=0 \\
& Q_{3}-Q_{5}=0 \\
& Q_{2}-Q_{4}=0 \\
& Q_{5}+Q_{4}-Q_{6}=0, \\
& P_{V_{1}}-P_{4}=0 \\
& P_{V_{2}}-P_{4}=0, \\
& P_{V_{3}}-P_{4}=0,
\end{aligned}
$$

where $c_{1}=\frac{A}{L}>0$ and $c_{2}=\frac{c_{f}}{2 D A \rho_{a}}>0$. For $t \geq t_{S}$ equations $(11 \mathrm{k}),(11 \mathrm{l}),(11 \mathrm{~m})$ will be replaced by

$$
Q_{4}=0, \quad Q_{5}=0, \quad Q_{6}=0 .
$$

In terms of the nonswitched DAE (10) we have

$$
\begin{aligned}
& E_{p}=\left[\begin{array}{ccccccccccccc}
-1 & 0 & 0 & 0 & 0 & 0 & 0 & 0 & 0 & 0 & 0 & 0 & 0 \\
0 & -1 & 0 & 0 & 0 & 0 & 0 & 0 & 0 & 0 & 0 & 0 & 0 \\
0 & 0 & -1 & 0 & 0 & 0 & 0 & 0 & 0 & 0 & 0 & 0 & 0 \\
0 & 0 & 0 & -1 & 0 & 0 & 0 & 0 & 0 & 0 & 0 & 0 & 0 \\
0 & 0 & 0 & 0 & -1 & 0 & 0 & 0 & 0 & 0 & 0 & 0 & 0 \\
0 & 0 & 0 & 0 & 0 & -1 & 0 & 0 & 0 & 0 & 0 & 0 & 0 \\
0 & 0 & 0 & 0 & 0 & 0 & 0 & 0 & 0 & 0 & 0 & 0 & 0 \\
0 & 0 & 0 & 0 & 0 & 0 & 0 & 0 & 0 & 0 & 0 & 0 & 0 \\
0 & 0 & 0 & 0 & 0 & 0 & 0 & 0 & 0 & 0 & 0 & 0 & 0 \\
0 & 0 & 0 & 0 & 0 & 0 & 0 & 0 & 0 & 0 & 0 & 0 & 0 \\
0 & 0 & 0 & 0 & 0 & 0 & 0 & 0 & 0 & 0 & 0 & 0 & 0 \\
0 & 0 & 0 & 0 & 0 & 0 & 0 & 0 & 0 & 0 & 0 & 0 & 0 \\
0 & 0 & 0 & 0 & 0 & 0 & 0 & 0 & 0 & 0 & 0 & 0 & 0
\end{array}\right], A_{p}=\left[\begin{array}{cccccccccccccc}
0 & 0 & 0 & 0 & 0 & 0 & c_{1} & 0 & 0 & 0 & 0 & 0 & 0 \\
0 & 0 & 0 & 0 & 0 & 0 & -c_{1} & c_{1} & 0 & 0 & 0 & 0 & 0 \\
0 & 0 & 0 & 0 & 0 & 0 & -c_{1} & 0 & c_{1} & 0 & 0 & 0 & 0 \\
0 & 0 & 0 & 0 & 0 & 0 & 0 & -c_{1} & 0 & 0 & c_{1} & 0 & 0 \\
0 & 0 & 0 & 0 & 0 & 0 & 0 & 0 & -c_{1} & 0 & 0 & c_{1} & 0 \\
0 & 0 & 0 & 0 & 0 & 0 & 0 & 0 & 0 & 0 & 0 & 0 & -c_{1} \\
1 & -1 & -1 & 0 & 0 & 0 & 0 & 0 & 0 & 0 & 0 & 0 & 0 \\
0 & 0 & 1 & 0 & -1 & 0 & 0 & 0 & 0 & 0 & 0 & 0 & 0 \\
0 & 1 & 0 & -1 & 0 & 0 & 0 & 0 & 0 & 0 & 0 & 0 & 0 \\
0 & 0 & 0 & 1 & 1 & -1 & 0 & 0 & 0 & 0 & 0 & 0 & 0 \\
0 & 0 & 0 & 0 & 0 & 0 & 0 & 0 & 0 & -s_{p} & s_{p} & 0 & 0 \\
0 & 0 & 0 & 0 & 0 & 0 & 0 & 0 & 0 & -s_{p} & 0 & s_{p} & 0 \\
0 & 0 & 0 & 0 & 0 & 0 & 0 & 0 & 0 & -s_{p} & 0 & 0 & s_{p}
\end{array}\right] \\
& f=\left(\begin{array}{c}
-P_{v_{R_{1}}} \\
0 \\
0 \\
0 \\
0 \\
P_{v_{R_{2}}} \\
0 \\
0 \\
0 \\
0 \\
0 \\
0 \\
0
\end{array}\right), \quad g_{p}(x)=\left(\begin{array}{c}
c_{2} Q_{1}\left|Q_{1}\right| \\
c_{2} Q_{2}\left|Q_{2}\right| \\
c_{2} Q_{3}\left|Q_{3}\right| \\
c_{2} Q_{4}\left|Q_{4}\right| \\
c_{2} Q_{5}\left|Q_{5}\right| \\
c_{2} Q_{6}\left|Q_{6}\right| \\
0 \\
0 \\
0 \\
0 \\
0 \\
0 \\
0
\end{array}\right),
\end{aligned}
$$


where $p=1,2$ and $s_{1}=1$ and $s_{2}=0$.

\section{Discussion on switched DAEs}

Note that the switched DAE (10) contains a nonlinear term $g_{\sigma}(x)$, therefore the distributional solution framework $[10,11]$ cannot be applied directly. Nonlinear switched DAEs were investigated in [9], but this approach excludes Dirac impulses in $x$ by definition, because if a Dirac impulse occurs in the solution $x$ of (10) (which we actually desire to capture the water hammer effect) then it is unclear how $g_{\sigma}(x)$ has to be evaluated in general (e.g. what is the sine of a Dirac impulse). Here we have a special structure which we can write in the following form

$$
\begin{gathered}
g(x)=\mathscr{N} \bar{g}(\mathscr{M} x), \\
\mathscr{M}=\left[\begin{array}{ll}
I_{\{6 \times 6\}} & \left.O_{\{6 \times 7\}}\right], \quad \mathscr{N}=\mathscr{M}^{\top} . \\
\overline{g_{i}}\left(Q_{i}\right)=-c_{2} Q_{i}\left|Q_{i}\right| \quad i=1, \cdots, 6 .
\end{array}\right.
\end{gathered}
$$

This special sturcture allows us to extend the distributional solution theory from the linear case to the nonlinear case, c.f. [7]. To keep it simple here, consider the individual equation $-\frac{d Q_{i}}{d t}=c_{1}\left(P_{v_{i}}-P_{v_{R_{1}}}\right)+c_{2} Q_{i}\left|Q_{i}\right|$ and let us denote by $Q_{i}\left(t_{s}^{-}\right)$, $Q_{i}\left(t_{s}^{+}\right)$the flow before and after the switching time $t_{s}$. When the valves are closed all flows become zero, in particular $Q_{i}\left(t_{s}^{+}\right)=0$ and since in general $Q_{i}\left(t_{s}^{-}\right) \neq 0$ there will be Dirac impulse in $\frac{d Q_{i}}{d t}$ at the switching time $t_{s}$. In fact, the impulse part of $\frac{d Q_{i}}{d t}$ at $t_{s}$ is given by

$$
\frac{d Q_{i}}{d t}\left[t_{s}\right]=Q_{i}\left(t_{s}^{+}\right)-Q_{i}\left(t_{s}^{-}\right) \delta_{t_{s}}=-Q_{i}\left(t_{s}^{-}\right) \delta_{s}
$$

and for $t>t_{s}$ we have $\frac{d Q_{i}}{d t}=0$ because $Q_{i}$ is identically zero. Altogether we can conclude from (11) together with (12) that for $t \geq t_{s}$ :

$$
\begin{aligned}
& P_{v_{1}}=\frac{1}{c_{1}} Q_{1}\left(t_{s}^{-}\right) \delta_{t_{s}}+P_{R_{1}}=\frac{1}{c_{1}} Q_{1}\left(t_{s}^{-}\right) \delta_{t_{s}}+P_{R_{1}}, \\
& P_{v_{2}}=\frac{1}{c_{1}} Q_{2}\left(t_{s}^{-}\right) \delta_{t_{s}}+P_{v_{1}}=\frac{1}{c_{1}}\left(Q_{2}\left(t_{s}^{-}\right)+Q_{1}\left(t_{s}^{-}\right)\right) \delta_{t_{s}}+P_{R_{1}}, \\
& P_{v_{3}}=\frac{1}{c_{1}} Q_{3}\left(t_{s}^{-}\right) \delta_{t_{s}}+P_{v_{1}}=\frac{1}{c_{1}}\left(Q_{3}\left(t_{s}^{-}\right)+Q_{1}\left(t_{s}^{-}\right)\right) \delta_{t_{s}}+P_{R_{1}}, \\
& P_{V_{1}}=\frac{1}{c_{1}} Q_{4}\left(t_{s}^{-}\right) \delta_{t_{s}}+P_{v_{2}}=\frac{1}{c_{1}}\left(Q_{4}\left(t_{s}^{-}\right)+Q_{3}\left(t_{s}^{-}\right)+Q_{1}\left(t_{s}^{-}\right)\right) \delta_{t_{s}}+P_{R_{1}}, \\
& P_{V_{2}}=\frac{1}{c_{1}} Q_{5}\left(t_{s}^{-}\right) \delta_{t_{s}}+P_{v_{3}}=\frac{1}{c_{1}}\left(Q_{5}\left(t_{s}^{-}\right)+Q_{3}\left(t_{s}^{-}\right)+Q_{1}\left(t_{s}^{-}\right)\right) \delta_{t_{s}}+P_{R_{1}}, \\
& P_{V_{3}}=\frac{1}{c_{1}} Q_{6}\left(t_{s}^{-}\right) \delta_{t_{s}}+P_{R_{2}}=\frac{1}{c_{1}} Q_{6}\left(t_{s}^{-}\right) \delta_{t_{s}}+P_{R_{2}} .
\end{aligned}
$$

The coefficient in front of $\delta_{t_{s}}$ determine the impulse length. For $t>t_{s}$ its clear that all pressures will settle down as 


$$
p_{v_{1}}=p_{v_{2}}=p_{v_{3}}=p_{V_{1}}=p_{V_{2}}=P_{R_{1}}, \quad p_{V_{3}}=P_{R_{2}} .
$$

\section{Comparison of both modeling approaches}

Our focus here is to observe the jump and Dirac impulse in the pressure, due to the instantaneous closure of valves located at $V_{1}, V_{2}$. In particular, we assume that the PDE solution on $\left[0, t_{S}\right)$ is stationary, i.e. $q_{i}(t, x) i=1, \cdots, 6$ is approximately constant in time and space (or in other words, when the valves are closed the dynamics in all pipe have approximately settled down). For the numerical simulations we use a first order Godunov scheme with artificial viscosity $(<0.25)$. Figure 3 shows the results for the pressure value at $V_{1}$ (similar plots result also for the pressure at $V_{2}$ ) over the time interval $[3 s, 8 s]$ with initial values

$$
q_{i}(0, x) \equiv 0, \quad \rho_{i}(0, x) \equiv 1 \times 10^{3}
$$

and pipes parameters:

$$
\begin{aligned}
& P_{a}=1.01 \times 10^{6}, \quad \beta=\frac{1}{K}=4 \times 10^{-9}, \quad \rho_{a}=1000, \\
& L=5, \quad D=0.5, \quad c_{f}=0.02 \text {. }
\end{aligned}
$$

We have chosen a moderate ratio between length and diameter of pipe, so that the water hammer effect is better visible. The parameters $P_{a}, \rho_{a}$ and $\beta$ are physical parameters and $c_{f}$ is chosen via the so-called moody chart, see e.g. [8].

Figure 3 clearly shows a strong pressure spike just after the switching time $t_{S}=4 s$, the pressure oscillatoryly settles to a new pressure value say $\bar{P}_{R}^{1}$. The same behavior occurs for $P_{V_{2}}$ which settles to $\bar{P}_{R}^{1}$. Instead of running the simulation for a very long time, we just chose a settling time $\varepsilon>0$ and take the average of the pressures on the interval $\left(t_{S}+\varepsilon, T\right]$ where $T>t_{S}+\varepsilon$ is our overall simulation time, i.e.

$$
\begin{aligned}
& \bar{P}_{R}^{1}:=\frac{1}{T-\left(t_{S}+\varepsilon\right)} \int_{t_{S}+\varepsilon}^{T} p_{V_{1}}(t, x) \mathrm{d} t \\
& \bar{P}_{R}^{2}:=\frac{1}{T-\left(t_{S}+\varepsilon\right)} \int_{t_{S}+\varepsilon}^{T} p_{V_{2}}(t, x) \mathrm{d} t .
\end{aligned}
$$

With

$$
\varepsilon=1.6, \quad T=8
$$

we obtain

$$
\bar{P}_{R}^{1} \approx \bar{P}_{R}^{2} \approx 8.23 \times 10^{8}
$$

The value predicted by the switched DAE solution for $t>t_{s}$ from (14) is,

$$
P_{v_{R_{1}}}\left(t_{S}^{+}\right)=P_{R_{1}} \approx 8.23 \times 10^{8}
$$



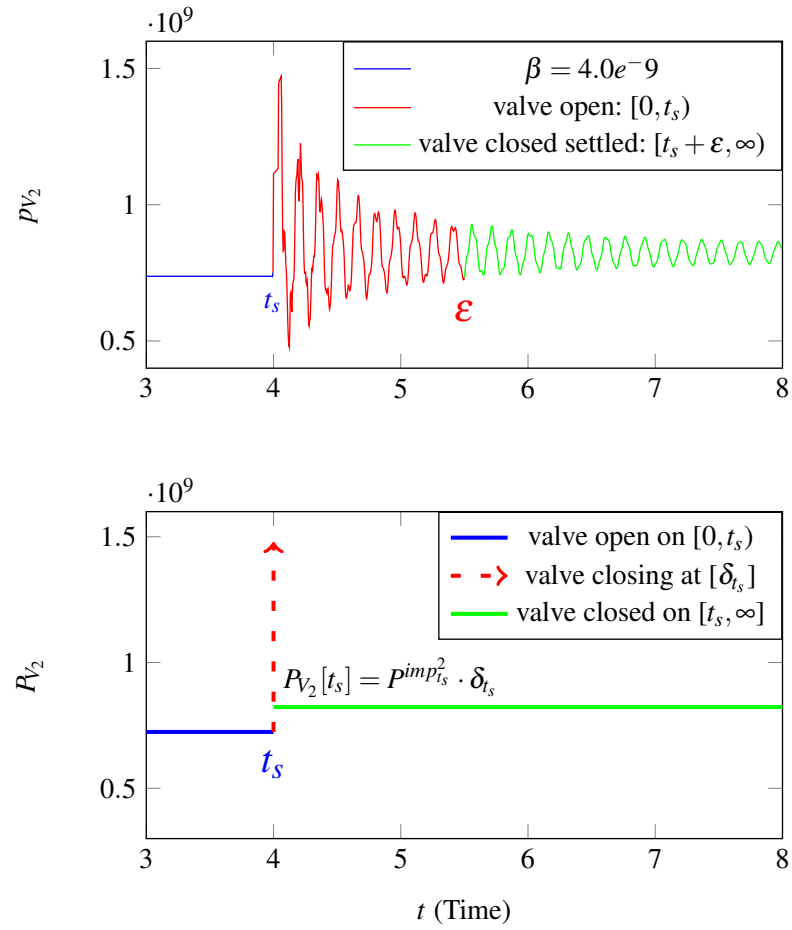

Fig. 3: Comparison of pressure profile PDE models $\left(p_{V_{2}}\right)$ (above) and switched DAE model $\left(P_{V_{2}}\right)$ (below), profile for $p_{V_{1}}$ is approximately symmetrical

In Table 1 the relative error between $\bar{P}_{R}^{i}, i=\{1,2\}$ and $P_{v_{R_{1}}}\left(t_{S}^{+}\right)$is presented for decreasing compressibility coefficients $\beta$.

\begin{tabular}{ccc|c|c}
\hline$\beta$ & $\bar{P}_{R}^{1}$ & $\bar{P}_{R}^{2}$ & $\frac{\bar{P}_{R}^{1}-P_{v_{R_{1}}}\left(t_{S}^{+}\right)}{P_{v_{R_{1}}}\left(t_{S}^{+}\right)}$ & $\left.\frac{\bar{P}_{R}^{2}-P_{v_{R_{1}}}\left(t_{S}^{+}\right)}{P_{v_{R_{1}}} t_{S}^{+}}\right)$ \\
\hline $15.0 \cdot 10^{-9}$ & $8.1613 \cdot 10^{8}$ & $8.2494 \cdot 10^{8}$ & $8.3 \cdot 10^{-03}$ & $2.4 \cdot 10^{-03}$ \\
$9.0 \cdot 10^{-9}$ & $8.2644 \cdot 10^{8}$ & $8.2419 \cdot 10^{8}$ & $4.2 \cdot 10^{-03}$ & $1.4 \cdot 10^{-03}$ \\
$4.0 \cdot 10^{-9}$ & $8.2401 \cdot 10^{8}$ & $8.2408 \cdot 10^{8}$ & $1.2 \cdot 10^{-03}$ & $1.3 \cdot 10^{-03}$ \\
$5.0 \cdot 10^{-10}$ & $8.2329 \cdot 10^{8}$ & $8.2352 \cdot 10^{8}$ & $3.5 \cdot 10^{-04}$ & $6.3 \cdot 10^{-04}$ \\
$2.0 \cdot 10^{-9}$ & $8.2317 \cdot 10^{8}$ & $8.2348 \cdot 10^{8}$ & $2.6 \cdot 10^{-04}$ & $5.8 \cdot 10^{-04}$ \\
\hline
\end{tabular}

Table 1: Comparison of pressure at valves $V_{1}$ and $V_{2}$ for PDE and switched DAE model.

In order to compare the peak in $P_{V_{1}}, P_{V_{2}}$ just after the valve is closed with the Dirac impulse $P_{V_{1}}\left[t_{S}\right]$ and $P_{V_{2}}\left[t_{S}\right]$ in response to the switching time, we recall that a 
Dirac impulse $\delta_{t_{s}}$ at $t_{s}>0$ can be approximated by a sequence of functions $t \mapsto \delta_{t_{s}}^{\varepsilon}(t)$ such that $\delta^{\varepsilon}(t)=0$ for $t \neq\left[t_{s}, t_{s}+\varepsilon\right]$ and $\int_{t_{s}}^{t_{s}+\varepsilon} \delta_{t_{s}}^{\varepsilon}(t) \mathrm{d} t=1$. We therefore make the Ansatz for $p_{V_{1}}$ and $P_{V_{2}}$,

$$
p_{V_{1}} \approx \bar{P}^{\mathrm{imp} t_{S}} \delta^{\varepsilon}(t)+\bar{P}_{R}^{1}, \quad p_{V_{2}} \approx \bar{P}^{\mathrm{imp} t_{S}^{2}} \delta^{\varepsilon}(t)+\bar{P}_{R}^{2} \quad t \in\left(t_{S}, T\right] .
$$

hence we can approximate the magnitude of the "smoothed-out" Dirac impulse occurring in the PDE model as follows:

$$
\bar{P}^{\mathrm{imp} t_{S}^{1}}:=\int_{t_{S}}^{t_{S}+\varepsilon} p_{V_{1}}-\bar{P}_{R}^{1} \mathrm{~d} t
$$

analougously for $p_{V_{2}}$,

$$
\bar{P}^{\mathrm{imp} t_{S}^{2}}:=\int_{t_{S}}^{t_{S}+\varepsilon} p_{V_{2}}-\bar{P}_{R}^{2} \mathrm{~d} t .
$$

The Dirac impulse induced by the switched DAE are defined from (14) i.e.,

$$
\begin{aligned}
& P_{V_{1}}\left[t_{S}\right]=\frac{1}{c_{1}}\left(Q_{4}\left(t_{s}^{-}\right)+Q_{3}\left(t_{s}^{-}\right)+Q_{1}\left(t_{s}^{-}\right)\right) \delta_{t_{s}}=: P^{\mathrm{imp}_{t_{S}}^{1}} \delta_{t_{s}}, \\
& P_{V_{2}}\left[t_{S}\right]=\frac{1}{c_{1}}\left(Q_{5}\left(t_{s}^{-}\right)+Q_{3}\left(t_{s}^{-}\right)+Q_{1}\left(t_{s}^{-}\right)\right) \delta_{t_{s}}=: P^{\mathrm{imp}_{t_{S}}^{2}} \delta_{t_{s}} .
\end{aligned}
$$

A comparison between $\bar{P}^{\mathrm{imp} t_{S}}$ with $P^{\mathrm{imp} t_{S}}$ and $\bar{P}^{\mathrm{imp} t_{S}}$ with $P^{\mathrm{imp} t_{S}}$ for different values of the compressibility coefficient $\beta$ is presented in Table 2. For large $\beta$ the approximation is not very accurate, however, for decreasing compressibility the accuracy of the approximation improves.

\begin{tabular}{ccccccc}
\hline$\beta$ & $\bar{P}^{\mathrm{imp}_{t_{S}}^{1}}$ & $\bar{P}^{\mathrm{imp}} P_{t_{S}}^{2}$ & $P^{\mathrm{imp}_{t_{S}}^{1}}$ & $P^{\mathrm{imp}_{t_{S}}^{2}}$ & $\frac{\left|\bar{P}^{\mathrm{imp}_{t_{S}}^{1}}-P^{\mathrm{imp}_{t_{S}}^{1}}\right|}{P^{\mathrm{imp}_{t_{S}}^{1}}}$ & $\frac{\bar{P}^{\mathrm{imp}_{S_{S}}^{2}}-P^{\mathrm{imp}_{t_{S}}^{2}}}{P^{\mathrm{imp}_{t_{S}}^{2}}}$ \\
\hline $15.0 \cdot 10^{-9}$ & $5.7821 \cdot 10^{7}$ & $5.7831 \cdot 10^{7}$ & $5.1137 \cdot 10^{7}$ & $5.1137 \cdot 10^{7}$ & 0.1307 & 0.1309 \\
$9.0 \cdot 10^{-9}$ & $3.3944 \cdot 10^{7}$ & $3.3951 \cdot 10^{7}$ & $3.8590 \cdot 10^{7}$ & $3.8590 \cdot 10^{7}$ & 0.1204 & 0.1202 \\
$4.0 \cdot 10^{-9}$ & $3.0906 \cdot 10^{7}$ & $3.0918 \cdot 10^{7}$ & $2.8407 \cdot 10^{7}$ & $2.8407 \cdot 10^{7}$ & 0.0880 & 0.0884 \\
$5.0 \cdot 10^{-10}$ & $2.0299 \cdot 10^{7}$ & $2.0292 \cdot 10^{7}$ & $2.1096 \cdot 10^{7}$ & $2.1096 \cdot 10^{7}$ & 0.0378 & 0.0381 \\
$2.0 \cdot 10^{-10}$ & $1.8450 \cdot 10^{7}$ & $1.8457 \cdot 10^{7}$ & $1.8482 \cdot 10^{7}$ & $1.8482 \cdot 10^{7}$ & 0.0017 & 0.0014 \\
\hline
\end{tabular}

Table 2: Impulse length comparison

Similar as for the PDE simulations we assume that the DAE is stationary before we switch, i.e. $Q_{i}\left(t_{S}^{-}\right)$for $i \in\{1, \cdots, 6\}$ before closing of the valve. It should be noted that although the compressibility coefficient $\beta$ does not effect the parameters of the switched DAE model, it does effect the initial value $q_{0}$ (and hence via 
$Q_{i}\left(t_{S}^{-}\right)$, because this is chosen to match the stationary solution of the balance law (8) considered on $\left[0, t_{S}\right)$ which depends on $\beta$.

\section{Conclusion}

We have presented a switched DAE model for water hammer on a simple setup, which we compared with a compressible nonlinear system of balance laws. With the support of numerical simulations of the PDE model we justified our conjecture that a switched DAE model is a good approximation for the PDE model with small compressibility coefficient. In future we will focus on a formal proof of convergence as well as the treatment of larger networks with asynchronously closed valves.

\section{Acknowledgement}

We are thankful to Jochen Kall for fruitful discussions concerning the PDE simulations of earlier versions of this work.

\section{References}

1. S. AdAmi, X. Y. Hu, AND N. A. AdAMS, Simulating three-dimensional turbulence with SPH, in Center for Turbulence Research, Proceedings of the Summer Program 2012, 2012, pp. 177-185.

2. M. H. ChAUDhrY AND L. MAYs, Computer modeling of free-surface and pressurized flows, Springer Science \& Business Media, 2012.

3. M. Herty, J. Mohring, AND V. SAChers, A new model for gas flow in pipe networks, Mathematical Methods in the Applied Sciences, 33 (2010), pp. 845-855.

4. J. IZQUIERDO, R. PÉREZ, AND P. L. IgLESIAS, Mathematical models and methods in the water industry, Mathematical and Computer Modelling, 39 (2004), pp. 1353-1374.

5. L. JANSEN AND J. PADE, Global unique solvability for a quasi-stationary water network model. Preprint series: Institut für Mathematik, Humboldt-Universität zu Berlin (ISSN 08630976), 2013-11, 2013.

6. L. JANSEN AND C. TISCHENDORF, A unified (P)DAE modeling approach for flow networks, in Progress in Differential-Algebraic Equations: Deskriptor 2013, S. Schöps, A. Bartel, M. Günther, W. E. J. ter Maten, and C. P. Müller, eds., Springer-Verlag, Berlin, Heidelberg, 2014, pp. 127-151.

7. J. KALL, R. KAUSAR, AND S. TREnN, Modeling water hammers via PDEs and switched DAEs with numerical justification. submitted for publication, 2016.

8. B. E. LAROCK, R. W. JEPPSON, AND G. Z. WATTERS, Hydraulics of pipeline systems, CRC press, 1999.

9. D. Liberzon AND S. TRENn, Switched nonlinear differential algebraic equations: Solution theory, Lyapunov functions, and stability, Automatica, 48 (2012), pp. 954-963.

10. S. Trenn, Distributional differential algebraic equations, $\mathrm{PhD}$ thesis, Institut für Mathematik, Technische Universität Ilmenau, Universitätsverlag Ilmenau, Germany, 2009.

11. —_, Regularity of distributional differential algebraic equations, Math. Control Signals Syst., 21 (2009), pp. 229-264.

12. _ـ Switched differential algebraic equations, in Dynamics and Control of Switched Electronic Systems - Advanced Perspectives for Modeling, Simulation and Control of Power Converters, F. Vasca and L. Iannelli, eds., Springer-Verlag, London, 2012, ch. 6, pp. 189-216.

13. E. B. Wylie AND V. L. Streeter, Fluid transients, McGraw-Hill International Book Co., New York, 1978. 